\section{Does an iridotomy provide protection against narrowing of the anterior chamber angle during Valsalva maneuvre in eyes with primary angle closure}

R Sihota', T Dada', A Aggarwal', G Srinivasan'1, V Gupta' and VK Chabra²

\begin{abstract}
Purpose To evaluate changes at the anterior chamber angle during Valsalva manoeuver, in eyes having primary angle closure (PAC) and a patent laser peripheral iridotomy.

Methods Twenty-three eyes of 23 consecutive patients underwent a recording of applanation tonometry, measurement of the anterior chamber angle recess, angle opening distance, iris thickness, anterior chamber depth, and pupil size on ultrasound biomicroscopy before and during the Valsalva maneuver. The Valsalva manoeuver was standardized to a pressure of $40 \mathrm{mmHg}$ for $15 \mathrm{~s}$, using a manometer.

Results The mean baseline intraocular pressure changed from $18.86+3.79$ to $26.73+4.73 \mathrm{mmHg}$ during Valsalva, $(P<0.0001)$. The anterior chamber angle recess narrowed from $16.62+6.24$ to $6.5+4.02$ degrees $(P<0.0001)$. There was a significant increase in the thickness of the ciliary body from $0.92+0.25$ to $1.17+0.31 \mathrm{~mm}(P=0.0006)$ and in the iris thickness from $0.47+0.17$ to $0.57+0.11 \mathrm{~mm}(P=0.007)$. A significant increase in pupillary diameter $(P=0.008)$ and a decrease in the angle opening distance $(P<0.0001)$ also occurred during Valsalva, whereas there was no significant change in the anterior chamber depth $(P=0.056)$. The angle recess during Valsalva had a positive correlation with the baseline anterior chamber angle $(r=0.41, P=0.05)$ and a negative correlation with the ciliary body thickness $(r=-0.52, P=0.046)$.
\end{abstract}

Conclusions The induction of Valsalva maneuver in day-to-day activities can lead to significant anterior segment angle shallowing and can lead to progression from the PAC stage to primary angle closure glaucoma in such predisposed eyes. The presence of a patent laser iridotomy may not prevent irido trabecular apposition during the Valsalva maneuver.

Eye (2008) 22, 389-393; doi:10.1038/sj.eye.6702646; published online 6 April 2007

Keywords: Valsalva; iridotomy; UBM; primary angle closure; primary angle closure glaucoma

\section{Introduction}

We frequently perform the Valsalva maneuver during normal daily activities, and this leads to various physiological changes such as a rise in blood pressure, increased intrathoracic pressure, increased peripheral venous pressure, and changes in the intraocular pressure (IOP) ${ }^{1-6}$ Although the effect of the Valsalva maneuver on intraocular pressure (IOP) in the normal population and a few glaucomatous eyes has been documented, ${ }^{3-6}$ its effect on the anterior chamber angle using ultrasound biomicroscopy was recently evaluated, and a significant narrowing of the angle recess was documented. ${ }^{7}$

This cross-sectional study was designed to evaluate the protective efficacy of a laser iridotomy on the effect produced by the Valsalva manoeuver in eyes with primary angle closure (PAC), using ultrasound biomicroscopy (UBM).
${ }^{1}$ Glaucoma Research Facility، Dr Rajendra Prasad Center for Ophthalmic Sciences, All India Institute of Medical Sciences, New Delhi, India

${ }^{2}$ Department of Biostatistics, All India Institute of Medical Sciences, New Delhi, India

Correspondence: T Dada, All India Institute of Medical Sciences,

Dr Rajendra Prasad Center for Ophthalmic Sciences, New Delhi, India Tel: + 911126589695 ; Fax: +911126588919 E-mail: tanujdada@ hotmail.com

Received: 23 June 2006 Accepted in revised form: 2 October 2006 Published online: 6 April 2007

This manuscript has not been published or presented anywhere 


\section{Materials and methods}

Consecutive, eligible patients aged between 40 and 60 years referred for glaucoma evaluation to the Glaucoma research facility, between January and December 2005, were enrolled in the study. The inclusion criteria were a clear cornea allowing accurate applanation tonometry and gonioscopy, and the clinical diagnosis of primary angle closure (PAC) in both eyes of the patient. The criteria for clinical diagnosis of PAC were as follows:

Patients with PAC had to have gonioscopically documented presence of peripheral anterior synechiae, clumping of pigment in an occludable angle, that is, one with a steep peripheral iris configuration, and an angle recess narrowed to less than $20^{\circ}$, with the trabecular meshwork not being visualized in more than $180^{\circ}$ of the angle. The optic disc did not have glaucomatous damage.

All patients had a patent iridotomy performed at least 6 weeks before inclusion in the study. The laser peripheral iridotomy was performed using an Nd-YAG (neodymiumyttrium-aluminium-garnet) laser set at 2-6 mJ. A crypt in the midperipheral iris was selected for the iridotomy site. All patients received $0.1 \%$

betamethasone eye drops (q.i.d) for 1 week in addition to $0.5 \%$ timolol maleate (b.d) for the same period. At the

time of performing the UBM measurements, all patients had IOP $<21 \mathrm{mmHg}$ and were not on any ocular hypotensive medication. Patients with a prior history of uveitis, trauma, intraocular surgery, diabetes, ischaemic heart disease, and patients who were on systemic medications that could alter their autonomic status were excluded. The study was approved by our institutional review board, and all participants in the study gave their informed consent in accordance with the Declaration of Helsinki Principles.

Patients underwent a detailed ocular examination, including best-corrected visual acuity, slit-lamp examination, applanation tonometry with Perkins, gonioscopy, and fundus evaluation with a $+90 \mathrm{D}$ lens and Humphrey 30-2 full threshold perimetry to reach a definitive clinical diagnosis.

For the Valsalva maneuvre, a mouth piece was attached to a manometer, so that airway pressure could be monitored. The patients were asked to blow into the mouthpiece using their thoracic, and not their cheek, muscles. Patients were asked to look at the manometer dial kept $1 \mathrm{~m}$ away and exhale into the mouthpiece, so that they were aware of the pressure generated. The Valsalva maneuvre was standardized to achieve a pressure of $40 \mathrm{mmHg}$, maintained for $15 \mathrm{~s}$.

Patients were placed supine, $0.5 \%$ proparacaine was instilled, and IOP was measured by a Perkin's applanation tonometer. The applanation IOP was measured $10 \mathrm{~s}$ after obtaining a pressure of $40 \mathrm{mmHg}$ on the manometer. Imaging of the anterior chamber and angle structures was performed on the ultrasound biomicroscope (UBM) with a $50 \mathrm{MHz}$ transducer probe (Paradigm Technologies, Bedford, MA, USA). A plastic scleral cup was inserted into the right eye and filled with $2 \%$ methyl cellulose. Only the right eye of each patient was included in the study. No pressure was applied with the scleral cup onto the globe while performing the UBM scan, as indentation by the scleral cup could distort the angle. Two baseline, standard, and axial UBM image sections of the superior angle and central anterior chamber were recorded. The entire procedure was conducted under standard lighting conditions, and variations in accommodation were minimized, as the patient was asked to maintain fixation at the manometer dial with the left eye.

The UBM scans taken before and after the procedure were given a code and transferred to a computer. The UBM images, pre- and post-Valsalva, were taken by a single trained ophthalmologist (TD). The image saved for measurement did not show the iridotomy site. The measurements were performed by another ophthalmologist (GV) who was masked to the study and was not aware of the Valsalva maneuver or whether the images was taken before or after any intervention. The following parameters were analyzed as described previously, by using the caliper system of the UBM machine: ${ }^{8}$

1. The anterior chamber angle recess was determined as follows. The apex of the peripheral irido-corneal angle was taken as the first point and two lines drawn from this point. The first line was parallel to the corneal endothelium and the second one parallel to the anterior surface of the iris. The angle formed between these two lines was taken as the angle recess.

2. The angle opening distance 500 (AOD 500) is the distance between the posterior corneal surface and the anterior iris surface measured on a line perpendicular to the trabecular meshwork at $500 \mu \mathrm{m}$ from the scleral spur.

3. The angle opening distance 250 (AOD 250) is the distance between the posterior corneal surface and the anterior iris surface measured on a line perpendicular to the trabecular meshwork at $250 \mu \mathrm{m}$ from the scleral spur.

4. The trabecular-ciliary process distance (TCPD) was measured on a line extending from the corneal endothelium at $500 \mu \mathrm{m}$ from the scleral spur perpendicularly through the iris to the ciliary processes.

5. Iris thickness (IT) was measured by a perpendicular line drawn at $500 \mu \mathrm{m}$ from the scleral spur. 


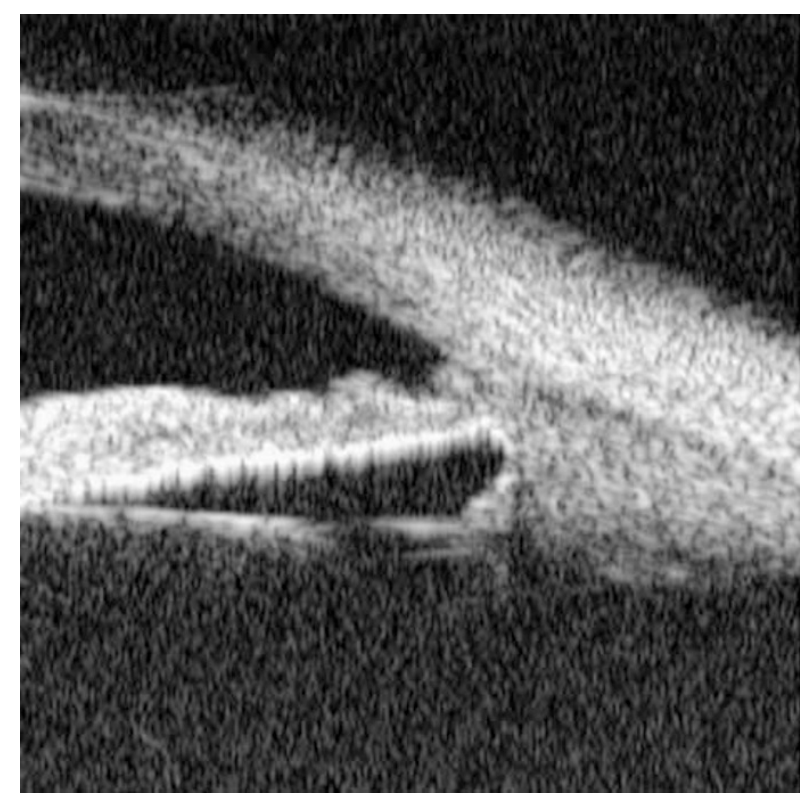

Figure 1 Wide open angle recess before Valsalva.

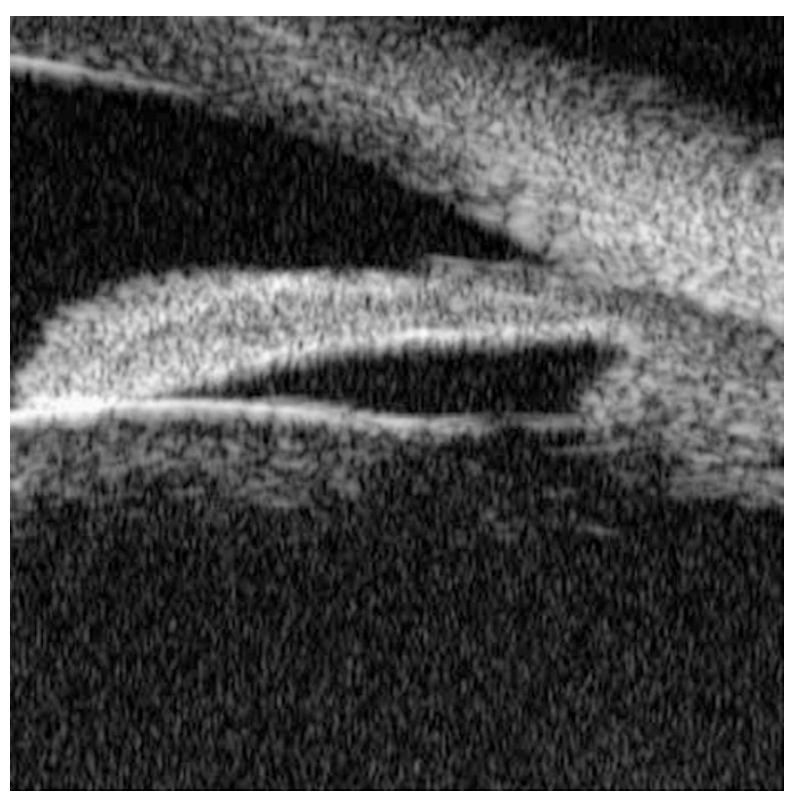

Figure 2 Narrowing of the angle recess with an increased convexity of the iris during Valsalva.

6. The ciliary body thickness (CBT) was measured along a line drawn from the most anterior point of attachment of the ciliary body to the sclera, across the anterior face of the ciliary body, to allow measurements from a fixed scleral point.

7. Central anterior chamber depth (ACD) was measured from the central corneal endothelium to the anterior capsule of the lens.
Table 1 IOP and anterior segment parameters before and during the Valsalva maneuvre

\begin{tabular}{lrrl}
\hline Anterior segment parameters & Baseline & $\begin{array}{c}\text { During } \\
\text { Valsalva }\end{array}$ & P-value \\
\hline IOP (mmHg) & $18.86 \pm 3.8$ & $26.73 \pm 4.73$ & 0.0001 \\
ARA (degree) & $16.62 \pm 6.24$ & $6.50 \pm 4.02$ & 0.0001 \\
AOD250 (mm) & $0.09 \pm 0.05$ & $0.05 \pm 0.05$ & 0.0001 \\
AOD500 (mm) & $0.18 \pm 0.08$ & $0.13 \pm 0.06$ & 0.0001 \\
Iris thickness (mm) & $0.47 \pm 0.17$ & $0.57 \pm 0.11$ & 0.0001 \\
Ciliary body thickness (mm) & $0.92 \pm 0.25$ & $1.17 \pm 0.31$ & 0.0006 \\
ACD (mm) & $2.27 \pm 0.21$ & $2.56 \pm 0.23$ & 0.056 \\
PD (mm) & $2.85 \pm 0.64$ & $2.96 \pm 0.58$ & 0.008 \\
\hline
\end{tabular}

Abbreviations: ACD, anterior chamber depth; AOD, angle opening distance; ARA, angle recess area; IOP, intraocular pressure; $\mathrm{PD}$, papillary diameter.

Values are mean $\pm \mathrm{SD}$

8. Pupil diameter (PD) was measured by using the caliper system within the UBM software.

For univariate analysis, a paired $t$-test was used to evaluate the significance of the difference in the various parameters before and after the Valsalva maneuvre. A multivariate linear regression analysis was carried out to identify factors contributing to changes at the anterior chamber angle recess, and the rise in IOP was recorded. Statistical analysis was performed using STATA version 7.0 program, and a $P$-value of less than 0.05 was taken as significant.

\section{Results}

Twenty-three eyes of 23 patients with PAC were evaluated. There were 11 men and 12 women. The mean age of the patients was $50.6+6.8$ years. There was a significant decrease in the anterior chamber angle recess (Figures 1 and 2), which narrowed from $16.62+6.24$ to $6.5+4.02^{\circ}(P<0.0001)$. Iridocorneal apposition was seen during Valsalva in 12 of the 23 eyes (52.2\%) in the superior angle. The AOD 250 decreased from $0.09 \pm 0.05$ to $0.05 \pm 0.05 \mathrm{~mm}(P<0.0001)$, whereas the AOD 500 decreased from $0.18 \pm 0.08$ to $0.13 \pm 0.06 \mathrm{~mm}(P<0.0001)$ during Valsalva.

There was a significant increase in the thickness of the ciliary body from $0.92+0.25$ to $1.17+0.31 \mathrm{~mm}$ $(P=0.0006)$, and from $0.47+0.17$ to $0.57+0.11 \mathrm{~mm}$ $(P=0.007)$ in the iris thickness. A significant increase in pupillary diameter (pupillary dilation) was noted with an increase from $2.85 \pm 0.64$ to $2.96 \pm 0.58 \mathrm{~mm}(P=0.008)$. There was a slight change in the anterior chamber depth from $2.27 \pm 0.21$ to $2.56 \pm 0.23 \mathrm{~mm}$, but this was not statistically significant $(P=0.056)$ (Table 1$)$.

The angle recess during Valsalva had a positive correlation with the baseline anterior chamber angle 
( $r=0.4107, P=0.05)$, and a negative correlation with the ciliary body thickness $(r=-0.52, P=0.046)$. The mean baseline IOP in the supine position before performing the Valsalva maneuvre was $18.86+3.79 \mathrm{mmHg}$, which increased to $26.73+4.73 \mathrm{mmHg}$ during the Valsalva $(P<0.0001)$.

\section{Discussion}

The Valsalva maneuvre comes into play during activities of daily living, and it is a well researched diagnostic tool for assessing the autonomic reflex control of the cardiovascular function. ${ }^{1,2}$ In studying this phenomenon, we decided to use a standard test that incorporated a form of breath holding.

There have been a few studies on the IOP changes induced by a Valsalva maneuvre. ${ }^{3-6}$ The UBM changes in the anterior chamber and anterior chamber angle recess during the Valsalva maneuvre were studied earlier, and showed a significant degree of narrowing of the angle with elevation of IOP during this maneuvre. ${ }^{7}$

In the present study, we studied PAC eyes, and found a rise of IOP, and a narrowing of the anterior chamber angle occurring despite the presence of a patent $\mathrm{Nd}$ :YAG iridotomy. All the eyes studied showed a significant narrowing, and appositional closure was seen in more than half the eyes. A narrowing of the angle was previously reported during Valsalva, ${ }^{7}$ leading to a significant narrowing of $<5^{\circ}$ in $60 \%$ of the eyes with a baseline angle of $<20^{\circ}$. Appositional closure was also seen in that study in one-third of the patients.

The rise of IOP in the eyes investigated by us was not surprising, as previous authors have seen this to occur in normal volunteers, but the rise of IOP was recorded to be associated with an increase in iris and ciliary body thickness. Venous stasis would also cause a simultaneous rise in episcleral venous pressure, the probable rationale for the rise in IOP. Dickerman et $a l^{4}$ postulated that the IOP rise was caused by increased retinal venous pressure. Lanigan et $a l^{5}$ thought that this rise of IOP was due to a systemic parasympathetic neuropathy.

An increased intrathoracic pressure would produce a venous stasis in these peripheral vessels, thickening the highly vascular ciliary body and iris. ${ }^{9-12}$ The significant iris root thickening recorded in our patients, probably another consequence of the venous stasis, could further narrow the angle recess. A similar venous stasis has been reported in secondary angle closure following a tight encirclage for retinal detachments. ${ }^{13}$

However, even the modest mydriasis encountered during the Valsalva maneuvre may contribute to an increase in the thickness at the iris root. We found that there was a pupillary dilation during the Valsalva maneuvre. A decreased vagal, and increased peripheral sympathetic stimulation has been reported during the Valsalva maneuvre, which could explain this dilation noted. ${ }^{14}$ Even this mild dilation could lead to further narrowing of the angle recess in predisposed eyes.

There was no significant shallowing of the central anterior chamber depth seen in our study. As this study was carried out with patients in the supine position, and measurements were made on the UBM picture, and not by ultrasound biometry, small changes in the anterior chamber depth may have been missed.

Primary angle closure glaucoma eyes have been shown to have a narrower anterior chamber depth on biometry, ${ }^{15}$ angle recess, and decreased trabecular-ciliary process distance as well as AOD as compared to normals. ${ }^{16}$ Gohdo et $\mathrm{al}^{17}$ found a thinning of the ciliary body in 18 normal eyes with narrow angles, which they found correlated to thickness of the lens and anterior chamber depth. The UBM changes recorded in the anterior segment in the present study are similar to those recorded during dark room or prone provocative tests for angle closure. An iris thickening and shortening has been reported by Pavlin. ${ }^{18}$ Narrowing of the angle with apposition has been noted in a number of studies. ${ }^{18,19}$ A convexity of the iris has also been frequently recorded.

UBM imaging of the anterior chamber angle during a Valsalva maneuvre could also be used to help identify eyes at risk for progression to primary angle closure glaucoma (PACG). PACG has been primarily ascribed to the occurrence of peripheral iridocorneal apposition or adhesions formed as a result of a relative pupillary block. However, there appear to be a large percentage of PACG eyes that do not have a history of such attacks or show evidence of a relative pupillary block and yet develop goniosynechiae or peripheral iris adhesions confined to the area of the trabecular meshwork pathomechanism of this chronic, progressive, 'creeping' angle closure is not known, and we hypothesize that this may be related to intermittent angle closure and a rise of IOP precipitated during activities, such as the Valsalva maneuvre, even in the presence of a patent iridotomy.

Repeated episodes of such apposition possibly occur in concert with other known precipitants of angle closure, such as mid-dilation of the pupil in twilight, and in situations of emotional stress. In predisposed eyes, prolonged iridocorneal apposition, in the presence of congestion of fenestrated vessels in the ciliary body, could lead to the formation of iridotrabecular or iridocorneal adhesions, and thus lead to progression of the disease process despite a patent laser iridotomy. This may be one of the reasons for progression from PAC to PACG despite an iridotomy.

A limitation of this study was the small sample size, the subjective element inherent in the measurement of the angle recess, and the posterior chamber angle. However, a 
certain degree of subjectivity remains in all UBM analysis, as the scleral spur has to be marked by an observer.

Ocular changes during the Valsalva maneuver are probably caused by the increased intrathoracic pressure, which causes a decreased venous return, together with a stimulation of the peripheral sympathetic system. The Valsalva maneuver commonly comes into play while lifting weights, straining during defecation and micturition, gagging, vomiting, blowing a stuffy nose, coughing, and using the arm or upper trunk muscles to move up in bed. The magnitude of the change in both the IOP and the anterior chamber angle, recorded on the UBM by us in similar circumstances, should be reason enough to warn all glaucoma patients, especially those with PACG to avoid or decrease such stressful activities, if at all possible.

The occurrence of a Valsalva maneuvre or similar physiological changes during activities of daily living may be responsible for intermittent angle closure in predisposed eyes as well as large, acute elevations of IOP which could be detrimental for a glaucomatous optic neuropathy, and may play a role in progression of the disease even after a laser iridotomy. Further longitudinal studies are required to establish the effect of the Valsalva maneuver on the anatomy of the peripheral irido-corneal angle in eyes with PAC after a laser iridotomy.

\section{References}

1 Porth C, Bamrah VS, Tristiani FE, Smith JJ. The Valsalva maneuver: mechanism and clinical implications. Heart Lung 1984; 13: 507-518.

2 Boothe R, Ryan J, Mellett H, Swiss E, Neth E. Hemodynamic changes associated with the Valsalva maneuver in normal men and women. J Lab Clin Med 1962; 59: 275-285.

3 Brody S, Erb C, Veit R, Rau H. Intraocular pressure changes: the influence of psychological stress and the valsalva manoeuvre. Biol Psychol 1999; 51: 43-57.

4 Dickerman RD, Smith GH, Langham-Roof L, McConathy WJ, East JW, Smith AB. IOP changes during maximal isometric contraction: does this reflect intracranial pressure or retinal venous pressure. Neurol Res 1999; 21: 243-246.

5 Lanigan LP, Clark CV, Allawi J, Hill DW, Keen H. Intraocular pressure responses to systemic autonomic stimulation in diabetes mellitus. Doc Ophthalmol 1989; 72: 141-153.
6 Rafuse PE, Mills DW, Hooper PL, Chang TS, Wolf R. Effects of Valsalva's maneuver on IOP. Can J Ophthalmol 1994; 29: 73-76.

7 Dada T, Gupta V, Deepak KK, Pandey RM. Narrowing of the anterior chamber angle during Valsalva maneuver: a possible mechanism of angle closure. Eur J Ophthalmol 2006; 16(1): 81-91.

8 Ishikawa H, Liebmann JM, Ritch R. Quantitative assessment of the anterior segment using ultrasound biomicroscopy. Curr Opin Ophthalmol 2000; 11: 133-139.

9 Friedman DS, Gazzard G, Foster P, Devereux JG, Broman A, Quigley $\mathrm{H}$ et al. Ultrasonographic biomicroscopy, Scheimflug photography and novel provocative tests in contralateral eyes of Chinese patients initially seen with acute angle closure glaucoma. Arch Ophthalmol 2003; 121: 633-642.

10 Kondo T, Miyazawa D, Unigame K, Kurimoto Y. Ultrasound biomicroscopic findings in humans with shallow anterior chamber and increased intraocular pressure after prone provocative test. Am J Ophthalmol 1997; 124: 632-640.

11 Sano R, Kurokawa T, Kurimoto Y, Miyazawa D, Yoshimura $\mathrm{N}$. Comparison between the anterior chamber configuration in the supine position and that in the prone position in patients with narrow angles. Nippon Ganka Gakkai Zasshi 2001; 105: 388-393.

12 Sakuma T, Sawada A, Yamamoto T, Kitazawa Y. Appositional angle closure in eyes with narrow angles: an ultrasound biomicroscopic study. J Glaucoma 1997; 6: 165-169.

13 Gedde SJ. Management of glaucoma after retinal detachment surgery. Curr Opin Ophthalmol 2002; 13: 103-109.

14 Marchini G, Pagliarusco A, Toscano A, Tosi R, Brunelli C, Bonomi L. Ultrasound biomicroscopic and conventional ultrasonographic study of ocular dimensions in primary angle closure glaucoma. Ophthalmology 1998; 105 2091-2098.

15 Sihota R, Gupta V, Agarwal HC, Pandey RM, Deepak KK Comparison of symptomatic and asymptomatic, chronic, primary angle-closure glaucoma, openangle glaucoma, and controls. J Glaucoma 2000; 9: 208-213.

16 Sihota R, Dada T, Gupta R, Lakshminarayan P, Pandey RM. Ultrasound biomicroscopy in the subtypes of primary angle closure glaucoma. J Glaucoma 2005; 14(5): 387-391.

17 Gohdo T, Tsumura T, Iijima H, Kashiwagi K, Tsukahara S. Ultrasound biomicroscopic study of ciliary body thickness in eyes with narrow angles. Am J Ophthalmol 2000; 129: 342-346.

18 Pavlin CJ, Harasiewicz K, Foster FS. An ultrasound biomicroscopic dark room provocative test. Ophthalmic Surg 1995; 26: 253-255.

19 Ishikawa H, Esaki k, liebmann JM, Uji Y, Ritch R. Ultrasound biomicroscopy dark room provocative testing: a quantitative method for estimating anterior chamber angle width. Jpn J Ophthalmol 1999; 43: 526-534. 